\title{
Design and Implementation of FPGA Based Environmental Protection Device for PCB Board
}

\author{
Zilong $\mathrm{Ma}^{1, \mathrm{a}}$, Jiaye $\mathrm{Mo}^{2, \mathrm{~b}}$ \\ ${ }^{1}$ Guangxi Technological College of Machinery and Electricity, Nanning 530007, China; \\ ${ }^{2}$ Nanning College for Vocational Technology, Nanning 530008, China.. \\ amazilong@163.com, b252614576 @qq.com
}

Keywords: FPGA, PCB, DS18B20, PH value, Corrosion machine.

\begin{abstract}
This paper introduces a kind of high efficiency, safety and low cost PCB board etching device which is suitable for the university laboratory, the scientific research unit and the small electronic enterprise based on FPGA. The system uses FPGA as the main controller, the temperature of the solution is measured by DS18B20, the concentration of the solution is monitored by the PH probe, the heating time can be set, the TFT touch screen is used as the man-machine interface, and the operation is simple. This system to replace sodium persulfate as ferric chloride etchant, not only clean and non-toxic, and has certain application value.
\end{abstract}

\section{Introduction}

The PCB board of electronic circuit is the design and practice of the course design, all kinds of competition and electronic innovation. It is the basic part of the electronic product development and the carrier of the electrical connection of the electronic components. The current technology of PCB system board is diverse, but in the teaching application, proof plate or stay in handmade plate. The corrosion inhibitor, ferric chloride used in the process of plate making, is a kind of harmful agent. The waste liquid produced by the plate making system not only pollutes the environment but also damages the health. This paper designs a set of FPGA as the main control, can detect the corrosion solution concentration, temperature, accurate heating, intelligent PCB environmental corrosion bubble catalytic system has the advantages of simple operation, reliable performance, easy to use, cost-effective, especially for the curriculum of electronic competition provides a good platform for design, plate making small batch PCB at any time, need the flexible production capacity [1].

\section{System Design}

\subsection{FPGA Selection.}

FPGA is a field programmable gate array device, which adopts the concept of logic cell array LCA design, and has different structure compared with PAL, GAL and other traditional logic circuits. It appears that the change of SCM in the micro control field dominance, FPGA programming language is a hardware description language, with parallel structure, faster than the speed of the programming language $C$ language this kind of order sentence structure, and is no longer involved in the interrupt concept can be realized at a time for.

This design will use the Cyclone IV series FPGA EPRCE10, the biggest advantage of the device is low power, nuclear voltage is only $1.2 \mathrm{~V}$, the 1032 logic unit contains $414 \mathrm{Kbit}$ embedded memory, 2 PLL, 179 user I/O, rich hardware and software resources, can well meet the design requirements.

\subsection{TFT Touch Screen.}

Amoy Shenzhen crystal TFT touch screen with Chi Electronics Co., TJC8048T070_011R, the screen is resistive touch screen, communication with USART serial interface, with a 7 inch display area, 16MB Flash, 2048Byte RAM, 3.3V (5V) interface level, 800*480 resolution. The screen will be the man-machine interface (HMI) of the underlying function of the package, through the serial port and the main control center for interactive communication. The screen supports 16 bit true color RGB 
display; supports text, buttons, sliders, progress bar, radio buttons, check boxes and other configuration control; support custom font support; drawing points, drawing lines, circles and so on GUI instruction; support BMP, JPG, PNG and other common image format; support simulator and hardware synchronous debugging. HMI serial port so that the development has become very simple, FPGA can easily communicate with the serial port, is very suitable for the development of the design requirements, greatly reducing the difficulty of writing the touch screen driver code.

\subsection{Device Structure.}

Small scale PCB plate making process is generally the first to draw the PCB file a circuit using CAD circuit software, then the PCB file to transfer paper printing, and then through the iron or heating machine thermal transfer paper on circuit transfer to the CCL, right after the clipping, chemical corrosion of CCL in rot etching solvent. The main content of our study is how fast, safe and environmentally friendly to the completion of the PCB copper clad corrosion.

PCB printed circuit board manufacturing process is mainly to achieve the removal of excess copper on the copper clad laminate [2]. The ferric chloride solution used in the past has strong corrosiveness, and the direct contact is harmful to the human body. We recommend selection of corrosion agent sodium persulfate environmental protection, it usually is white powder, soluble in water after the colorless, corrosion with copper ion generation copper blue reaction. The chemical formula of sodium persulfate corrosion PCB board as follows:

$\mathrm{Na} 2 \mathrm{~S} 2 \mathrm{O} 8+\mathrm{Cu}=\mathrm{Na} 2 \mathrm{SO} 4+\mathrm{CuSO} 4$

At first no corrosion of CCL, sodium persulfate solution is colorless, but with the process of corrosion, the solution turns blue, also means more consumption of sodium persulfate. 1:4 is the best proportion of environmental corrosive and water, if there is no concentration test, personal feeling ingredients, or is the beginning of proportion, but with the increase of the concentration of corrosion plate, corrosion speed will slow down. In this design, the PH probe is added (as shown in Figure 1), and the concentration of the corrosion solution can be detected at any time, which will increase the corrosion dose beyond the appropriate range. Sodium persulfate corrosion of copper the best temperature is 50 to $60 \mathrm{DEG} \mathrm{C}$, our device in both the heating rod, and check the multi-point temperature, so as to ensure the temperature of corrosion solution is stable in the temperature range. PCB plate corrosion device at the bottom of the increase of two strong air pump, the purpose is in the etching period, continue to produce bubbles, stirring corrosion liquid, accelerate the corrosion of PCB plate.

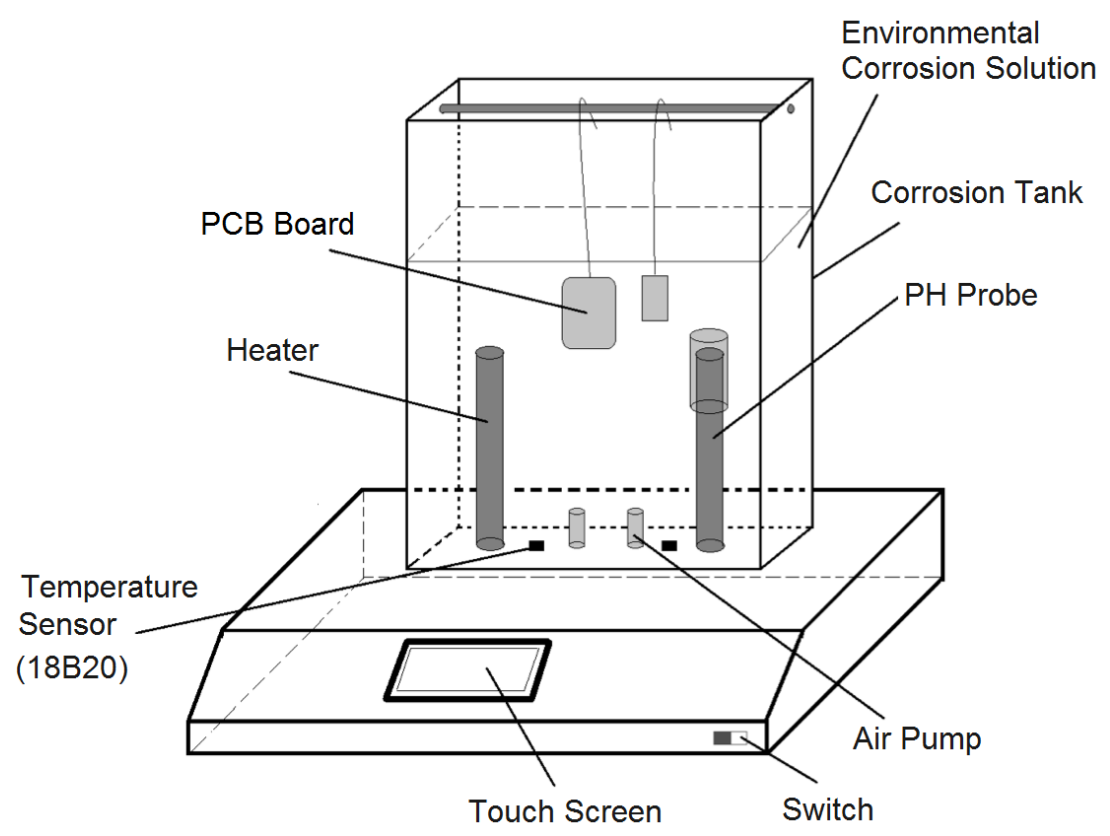

Fig. 1 Device model 


\subsection{System Design.}

This system takes FPGA as the control core, including the TFT touch screen, the temperature sensor, the $\mathrm{PH}$ probe sensor, the sound and light alarm circuit, the air pump control and the heating rod control and so on the function module, the system constitutes the block diagram as shown in figure 2.

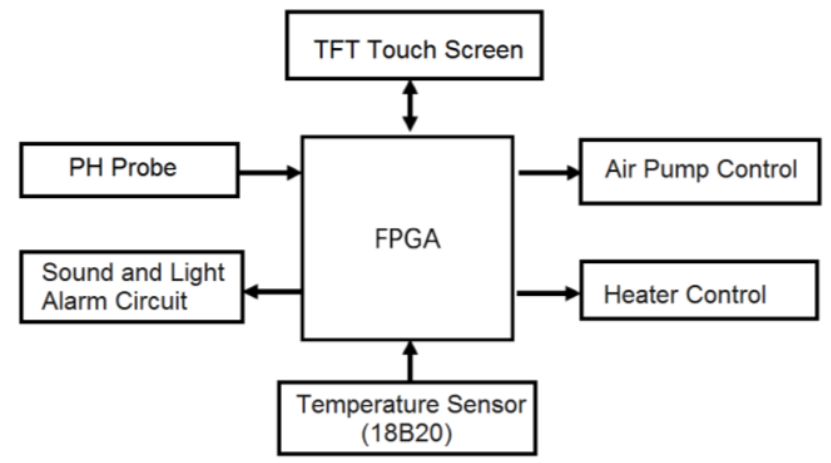

Fig. 2 System Block Diagram

Workflow description: because the system uses a touch screen, man-machine interface is very friendly, saving the physical buttons. Open the power switch, power system initialization, can through the touch screen solution temperature, pump intensity (high, medium and low grades) and etching time on the touch screen; then press the start button, the heating rod heating pump began to bubble stirred solution corrosion; corrosion solution temperature real-time detection of temperature don't set the temperature sensor, the heating range can reach, if the temperature control range, sound and light alarm circuit work and give tips, and stop heating, pump the whole process according to the set the intensity of work, until the time to set up the corrosion. PCB plate corrosion process is clearly visible, as long as the press pause button, midway can be stopped, and then press once to restore corrosion. After the corrosion time, the system will send out sound and light alarm prompt. PH probe sensor in the corrosion process, real-time monitoring of the $\mathrm{pH}$ value of the solution, if the acid increases, reaching a certain value, then the sound and light alarm to add corrosion agent powder to improve the solution concentration.

That is, although the corrosive agent itself harmless, but because of containing copper ions in wastewater, have impact on the environment, so the best treatment before disposal or lime soda and other alkaline substances in order to discard, protect the environment is to protect themselves.

\section{Software Design}

The design of the choice of the FPGA rather than the microcontroller as the main control system of the main reason lies in the FPGA programming language VHDL in the data processing more advantages. VHDL programming language - $\mathrm{C}$ language is a sequential language, and the language is the hardware description language, and contains a parallel structure, a variety of processes (events) can be run in parallel, the speed advantage is obvious. The system control program of simple, mainly consists of the following parts: TFT touch screen driver, DS18B20 driver, PH driver, probe the acousto-optic alarm program, air pump and a heating rod initialization program and control program, main program.

Touch the LCD screen can not only show the set time, corrosion the remaining time, set the temperature, the current temperature and $\mathrm{PH}$ value, you can also choose the pump stalls, hot rod heating state. In the interface directly integrated $4 \times 4$ matrix keyboard, system startup and stop button, save the physical button. FPGA through the serial port to achieve and touch the LCD screen data communication, as long as the instructions in accordance with the instruction set to send instructions, the screen will have a corresponding response, very convenient. 


\section{Summary}

The design of FPGA technology, touch screen technology and teaching and research combined to design and achieve an efficient, safe and low cost PCB board etching device, to solve the colleges and universities and scientific research units of small batch corrosion plate process problems, environmental and efficient, Has a certain practical value.

\section{Acknowledgments}

This paper was funded by The Scientific and Technological Research Project of Universities in 2015 (KY2015LX686) and Guangxi Institute of Mechanical and Electricity Engineering Project (2015KYZZ001).

\section{References}

[1]. Yugeng Zhang, Jin Wang. Design of PCB Board Carving Machine Based on AVR. Electronic Design Engineering.Vol. 6(2011), p. 143-145.

[2]. Lei Wei, Hui Zeng, et al. Design and Implementation of PCB Board Corrosion Device Based on AT89S52 MCU. Industrial Control Computer. Vol. 9(2016), p. 154-155. 